\title{
A Total and Convenient Synthesis of Orixiarine
}

\author{
T. Suresh ${ }^{1}$, K. Velmurugan ${ }^{2}$, P. S. Mohan ${ }^{1}$, R. Nandhakumar ${ }^{2 *}$ \\ ${ }^{1}$ Department of Chemistry, Bharathiar University, Coimbatore, India \\ ${ }^{2}$ Department of Chemistry, Karunya University, Karunya Nagar, Coimbatore, India \\ Email: "nandhakumar@karunya.edu
}

Received January 9, 2013; revised February 10, 2013; accepted February 28, 2013

\begin{abstract}
A simple total synthesis of the quinoline alkaloid, orixiarine was achieved from N-methylaniline and 1-bromo-3methyl-2-butanone.
\end{abstract}

Keywords: N-Methylaniline; 1-Bromo-3-methyl-2-butanone; Orixiarine; Total Synthesis

\section{Introduction}

In the recent past, a hemiterpenoid quinoline alkaloid, named orixiarine (Graph 1) was isolated from Skimmia laureola (Rutaceae), which is an aromatic gregarious evergreen shrub found in Western Himalayas and Kashmir $[1,2]$. The plant is used for the treatment of smallpox [3] and as an insecticide for domestic animals [4,5].

Earlier, our program of synthesis of quinoline alkaloids, in particular the hemiterpenoid quinoline alkaloid [6] was achieved with a multi-step protocol and in comparatively less yield. However, we aimed of providing a high yield procedure with significantly less steps, which also pave a path for deriving various alkaloids like edulinine, N-methylflindersine etc. [7]. Herein we report our investigations which have resulted in a four-step, convenient total synthesis of orixiarine starting from $\mathrm{N}$-methylaniline (2). When 4-hydroxy-N-methyl-2-quinolinone (3) $[8,9]$ was stirred with 1-bromo-3-methyl-2butanone (4) [10] in $4 \%$ sodium hydroxide solution gave $5(85 \%)$ along with a minor product $6(7 \%)$ under nucleophilic conditions and subsequent elimination of hydrogenbromide. Compound 5 on treatment with p-methyltoluenesulphonate and potassium carbonate yielded the final product 1 which on analysis by ${ }^{1} \mathrm{H}-\mathrm{NMR},{ }^{13} \mathrm{C}-\mathrm{NMR}$ and mass spectra, is found to be identical with that isolated from Skimmia laureola [1] (Scheme 1).

\section{Experimental}

Thin layer chromatography was used to access the reactions and the purity of products. Melting Points were determined on a Boetius Microheating Table and Mettler-FP5 Melting apparatus and are uncorrected. IR spectra were recorded in Shimadzu-8201FT instrument in

${ }^{*}$ Corresponding author.
$\mathrm{KBr}$ disc and only noteworthy absorption levels (reciprocal centimeter) are listed. ${ }^{1} \mathrm{H}-\mathrm{NMR}$ spectra were recorded in a AMX-400 $\mathrm{MHz}$ spectrometer in $\mathrm{CDCl}_{3}$ solution; chemical shifts are expressed in ppm $(\delta)$ relative TMS, coupling constants (J) in $\mathrm{Hz} .{ }^{13} \mathrm{C}-\mathrm{NMR}$ was also recorded on the same AMX-400 MHz spectrometer with Tetra methyl silane (TMS) as internal standard. Mass spectra were recorded on a Jeol-D-300 mass spectrometer. CHN analyses were carried out on a Carlo Erba 106 and Perkin-Elmer Model 240 analysers.

\section{Typical Procedures}

Preparation of 4-hydroxy-1-methylquinolin-2-one (3): 4-Hydroxy-1-methylquinolin-2-one (3) was prepared by reported procedure $[8,9]$.

Preparation of 1-bromo-3-methyl-2-butanone (4): A solution of 3-methyl-2-butanone $(8.6 \mathrm{~g}, 10.5 \mathrm{~mL}, 0.01$ mol) and $60 \mathrm{~mL}$ of anhydrous methanol was stirred and cooled in an ice-salt bath from $0^{\circ} \mathrm{C}-5^{\circ} \mathrm{C}$ and $5.46 \mathrm{~mL}$ $(0.01 \mathrm{~mol})$ of bromine was added in a rapid steady stream from the dropping funnel. During this time, the temperature was allowed to rise but was not permitted to exceed $10^{\circ} \mathrm{C}$. The reaction temperature was then maintained at $10^{\circ} \mathrm{C}$ throughout the remaining reaction time. The red colour of the solution faded gradually in about $45 \mathrm{~min}$, $30 \mathrm{~mL}$ of water was then added and the mixture was stirred at room temperature overnight. To this solution,<smiles>COc1c(CC(=O)C(C)C)c(=O)n(C)c2ccccc12</smiles>

Graph 1. Orixiarine. 


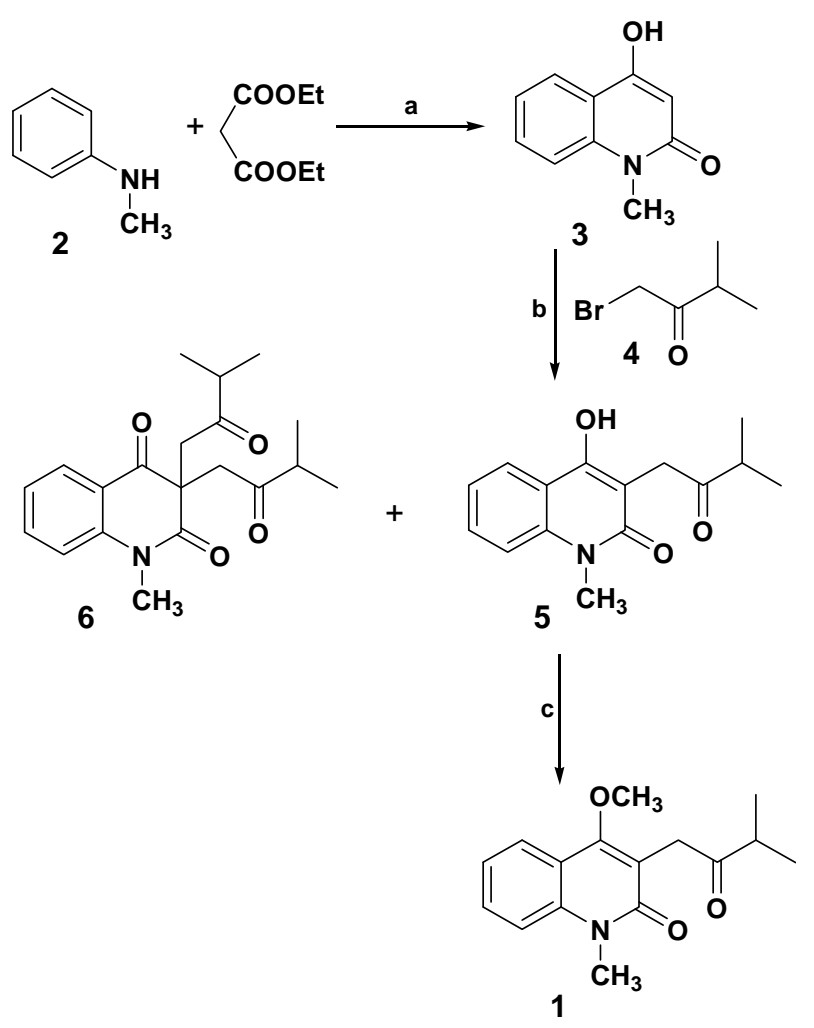

Scheme 1. Reagents \& Conditions: (a) $\mathrm{Ph}_{2} \mathrm{O}$, reflux, 5 h (85\%); (b) $4 \% \mathrm{NaOH}$, stirring, $70^{\circ} \mathrm{C}-80^{\circ} \mathrm{C}$ (85\%); (c) MPTSA, DMF, $\mathrm{K}_{2} \mathrm{CO}_{3}$, RT, stirring, 8 h (80\%)

$90 \mathrm{~mL}$ of water was added and the mixture was washed with four little portion of ether. The ether layers were combined and washed with $20 \mathrm{~mL}$ of aq $10 \%$ potassium carbonate and then twice with $20 \mathrm{~mL}$ portions of water. The ether layer was dried for 15 min over a few gram of sodium sulfate and the ether was evaporated to get 1-bromo-3-methyl-2-butanone. (yield: $10.0 \mathrm{~g}, 6.8 \mathrm{~mL}$, bp $83^{\circ} \mathrm{C}-85^{\circ} \mathrm{C}$ ) [10].

Preparation of 4-hydroxy-1-methyl-3-(3'-methyl-2'oxobutyl)-quinolin-2-one (5): 4-Hydroxy-1-methylquinolin-2-one (3) (0.700 g, $0.004 \mathrm{~mol})$ and 1-bromo-3methyl-2 butanone (4) $(0.66 \mathrm{~mL}, 0.004 \mathrm{~mol})$ was stirred in $4 \%$ sodium hydroxide solution at $70^{\circ} \mathrm{C}-80^{\circ} \mathrm{C}$ for $7-8$ $\mathrm{hr}$. The solid separated from the reaction mixture was filtered, dried and separated by column chromatography using pet. ether-ethyl acetate $(95: 5)$ which gave two products. Specific details on each product (5 and 6) are as follows.

Data for 5: $\mathrm{mp} 234^{\circ} \mathrm{C}$; yield $85 \%$; IR ( $\left.\mathrm{KBr}, \gamma \max \right)$ $1618,1680,3350 \mathrm{~cm}^{-1}$. ${ }^{1} \mathrm{H}-\mathrm{NMR}\left(\mathrm{CDCl}_{3}, 400 \mathrm{MHz}\right) \delta$ $1.0\left(\mathrm{~d}, 3 \mathrm{H}, \mathrm{CH}_{3}, \mathrm{~J}=6.02 \mathrm{~Hz}\right), \delta 1.2\left(\mathrm{~d}, 3 \mathrm{H}, \mathrm{CH}_{3}, 6.02\right.$ $\mathrm{Hz}), \delta 2.8\left(\mathrm{~m}, 1 \mathrm{H},-\mathrm{CH}-\left(\mathrm{CH}_{3}\right)_{2}\right), \delta 3.6\left(\mathrm{~s}, 3 \mathrm{H}, \mathrm{N}-\mathrm{CH}_{3}\right), \delta$ $3.8\left(\mathrm{~s}, 2 \mathrm{H}, \mathrm{CH}_{2}\right), \delta 7.28\left(\mathrm{dd}, 1 \mathrm{H}, \mathrm{C}_{6}-\mathrm{H}, \mathrm{J}=7.84 \mathrm{~Hz}\right), \delta$ $7.7\left(\mathrm{~d}, 1 \mathrm{H}, \mathrm{C}_{5}-\mathrm{H}, \mathrm{J}=6.02 \mathrm{~Hz}\right), \delta 7.9\left(\mathrm{dd}, 1 \mathrm{H}, \mathrm{C}_{7}-\mathrm{H}, \mathrm{J}=\right.$ $7.84 \mathrm{~Hz}), \delta 8.15\left(\mathrm{~d}, 1 \mathrm{H}, \mathrm{C}_{8}-\mathrm{H}, \mathrm{J}=6.02 \mathrm{~Hz}\right), \delta 12.5(\mathrm{~s}, 1 \mathrm{H}$, -OH). ${ }^{13} \mathrm{C} \mathrm{NMR}\left(\mathrm{CDCl}_{3}, 500 \mathrm{MHz}\right): 207,174.3,162.1$,
161.8, 141, 135, 126, 122.8, 122.4, 115, 114, 106, 42.3, 31.6, 30, 29. MS [70 eV, m/z $\left.\left(\mathrm{M}^{+}\right)\right]$259; Anal. Calcd for $\mathrm{C}_{15} \mathrm{H}_{17} \mathrm{NO}_{3}: \mathrm{C}, 69.46 ; \mathrm{H}, 6.61 ; \mathrm{N}, 5.41$ Found: C, 69.41; H, 6.55; N, 5.34 .

Data for 6: $\mathrm{mp} 170^{\circ} \mathrm{C}$; yield $7 \%$; IR ( $\left.\mathrm{KBr}, \gamma \max \right)$ 1672, 1635, $1610 \mathrm{~cm}^{-1}$. ${ }^{1} \mathrm{H}-\mathrm{NMR}\left(\mathrm{CDCl}_{3}, 400 \mathrm{MHz}\right) \delta$ $1.00(6 \mathrm{H}, \mathrm{m}), 1.2(6 \mathrm{H}, \mathrm{m}), 3.6(2 \mathrm{H}, \mathrm{h}, \mathrm{J}=7.37 \mathrm{~Hz}), 3.4$ $(4 \mathrm{H}, \mathrm{s}), 3.4(3 \mathrm{H}, \mathrm{s}), 7.25(1 \mathrm{H}, \mathrm{t}, \mathrm{J}=7.64 \mathrm{~Hz}), 7.6(1 \mathrm{H}, \mathrm{d}$, $\mathrm{J}=8.22 \mathrm{~Hz}), 7.8(1 \mathrm{H}, \mathrm{t}, \mathrm{J}=7.60 \mathrm{~Hz}), 8.00(1 \mathrm{H}, \mathrm{d}, \mathrm{J}=$ 7.64 Hz). Anal. Calcd for $\mathrm{C}_{20} \mathrm{H}_{25} \mathrm{NO}_{4}: \mathrm{C}, 69.95 ; \mathrm{H}, 7.34$; N, 4.08 Found: C, 69.45; H, 7.66; N, 4.14.

Synthesis of 4-methoxy-1-methyl-3-(3'-methyl-2'oxobutyl)-quinolin-2-one [Orixiarine] (1) 4-Hydroxy1-methyl-3-(3'-methyl-2'-oxobutyl)-quinolin-2-one (5) $(0.259 \mathrm{~g}, 0.001 \mathrm{~mol})$ was stirred with $p$-methyltoluenesulphonate $(0.186 \mathrm{~mL} .0 .001 \mathrm{~mol})$ and $\mathrm{K}_{2} \mathrm{CO}_{3}(250 \mathrm{mg})$ at room temperature for $6-8 \mathrm{hr}$. After completion of reaction, monitored by tlc, it was poured into crushed ice and allowed to stand overnight. The mixture was then extracted using ethyl acetate and the extract was dried over anhydrous sodium sulfate. Purification by silica gel column chromatography (pet. ether/EtOAc) yielded the product 1 as brownish powder. Yield $80 \%$; mp. $173^{\circ} \mathrm{C}$ $178^{\circ} \mathrm{C}$; IR $\left(\mathrm{KBr}, v_{\max }\right)-1665,1630 \mathrm{~cm}^{-1}{ }^{1} \mathrm{H}$ NMR $\left(\mathrm{CDCl}_{3}\right)[\delta \mathrm{ppm}]-1.2\left(\mathrm{~d}, 3 \mathrm{H}, \mathrm{CH}_{3}, \mathrm{~J}=6.45 \mathrm{~Hz}\right), 1.31(\mathrm{~d}$, $\left.3 \mathrm{H}, \mathrm{CH}_{3}, \mathrm{~J}=6.45 \mathrm{~Hz}\right), 2.8\left(\mathrm{~m}, 1 \mathrm{H}, \mathrm{CH}-\left(\mathrm{CH}_{3}\right)_{2}\right), 3.6(\mathrm{~s}$, $\left.3 \mathrm{H}, \mathrm{N}-\mathrm{CH}_{3}\right), 3.7\left(\mathrm{~s}, 3 \mathrm{H}, \mathrm{OCH}_{3}\right), 3.9\left(\mathrm{~s}, 2 \mathrm{H}, \mathrm{CH}_{2}\right), 7.28$ $\left(\mathrm{dd}, 1 \mathrm{H}, \mathrm{C}_{6}-\mathrm{H}, \mathrm{J}=5.82 \mathrm{~Hz}\right), 7.35\left(\mathrm{~d}, 1 \mathrm{H}, \mathrm{C}_{5}-\mathrm{H}, \mathrm{J}=5.34\right.$ $\mathrm{Hz}), 7.64$ (dd, 1H, $\left.\mathrm{C}_{7}-\mathrm{H}, \mathrm{J}=5.82 \mathrm{~Hz}\right), 8.0$ (d, 1H, $\mathrm{C}_{8}-\mathrm{H}, \mathrm{J}$ $=5.34 \mathrm{~Hz}) .{ }^{13} \mathrm{C} \mathrm{NMR}\left(\mathrm{CDCl}_{3}, 500 \mathrm{MHz}\right)-210.65,168.91$, $162.3,140.6,138,123,122.6,116,115,113,52.25,40.18$, 38, 31.58, 30.4, and 17.48. MS (m/z)-273. CHN Analysis (\%)-Calcd: C 70.31, H 7.01, N 5.12. $\left(\mathrm{C}_{16} \mathrm{H}_{19} \mathrm{NO}_{3}\right)$ Found: C 70.18, H 6.89, N 5.21.

\section{Conclusion}

In conclusion, a simple synthesis of the hemiterpenoid quinoline alkaloid, orixiarine was achieved and characterized. This paves the path for further exploration of the compound towards various potential biological studies.

\section{Acknowledgements}

TS thanks CSIR, New Delhi for the award of Senior Research Fellowship, PSM thanks CSIR-New Delhi for financial assistance (CSIR-Project). Authors thank SIF, IISc, Bangalore and IICT, Hyderabad for providing the spectral and analytical data.

\section{REFERENCES}

[1] Atta-ur-Rahman, M. N. Sultana, I. Choudhary, M. D. Shah and M. R. Khan, Journal of Natural Products, Vol. 61, 1998, p. 713.

[2] J. P. Michael, "Quinoline, Quinazoline and Acridone 
Alkaloids," Natural Product Reports, Vol. 20, No. 5, 2003, pp. 476-493. doi:10.1039/b208140g

[3] K. M. Nadkarni, "Indian Materia Medica," Popular Prakashan, Bombay, Vol. 1, 1976, p. 1142.

[4] T. Noshita, M. Tando, K. Suzuki, K. Murata and S. Funayama, Bioscience, Biotechnology, and Biochemistry, Vol. 65, 2001, p. 710.

[5] T. Namba, "Coloured Ilustrations of Wakan-yaku (The crude Drugs in Japan, China and the Neighbouring countries)," Vol. 1 (in Japanese), Hoikusha Publishing Co. Ltd., Osaka, 1980, p. 148.

[6] R. Nandhakumar, S. Thamarai Selvi, T. Suresh and P. S. Mohan, "A Synthesis of Orixiarine," Heterocycles, Vol. 57, No. 2, 2002, pp. 357-360. doi:10.3987/COM-01-9413

[7] M. F. Grundon, "The Alkaloids," Vol. 32, Academic press, London, 1988, p. 341

[8] L. A. Mitscher, G. W. Clark, T. Suzuki and M. S. Bathala, "A New Synthesis of Quinol-2,4-diones," Heterocycles, Vol. 3, No. 11, 1975, pp. 913-919. doi:10.3987/R-1975-11-0913

[9] P. Roschger and W. Stadlbaner, "Organic Azides in Heterocyclic Synthesis, 11. Ring Closure of 3-Acetyl-4azido-2-quinolones to Isoxazolo[4,3-c]quinolones," Liebigs Annalen der Chemie, Vol. 8, 1990, pp. 821-823. doi:10.1002/jlac.1990199001153

[10] R. A. Corral, O. O. Orazi and J. C. Autino, "Total Synthesis of Phenolic Balfourodendron Alkaloids," Tetrahedron Letters, Vol. 24, No. 23, 1983, pp. 2359-2360. doi:10.1016/S0040-4039(00)81924-3 